\title{
Dosimetric evaluation of three commercial radiotherapy planning systems for lung cancer and nasopharyngeal carcinoma cases
}

\section{Wan-jia Zheng}

Southern Theater Air Force Hospitial of the People's Liberation Army

Ming-li Wang

Sun Yat-sen University Cancer Center

Jun Zhang

Sun Yat-sen University Cancer Center

Yi-mei Liu

Sun Yat-sen University Cancer Center

Li Chen

Sun Yat-sen University Cancer Center

Xin YANG ( $\nabla$ yangxin@sysucc.org.cn )

Sun Yat-sen University Cancer Center https://orcid.org/0000-0001-7864-8518

\section{Si-juan Huang}

Sun Yat-sen University Cancer Center

\section{Research}

Keywords: Lung Cancer, Nasopharyngeal Carcinoma, Helical Tomotherapy, VMAT, IMRT, Dosimetric Evaluation

Posted Date: July 20th, 2020

DOI: https://doi.org/10.21203/rs.3.rs-29559/v2

License: (c) (1) This work is licensed under a Creative Commons Attribution 4.0 International License. Read Full License 
The authors have withdrawn this preprint from Research Square 